\title{
Mesenchymal phenotype after chemotherapy is associated with chemoresistance and poor clinical outcome in esophageal cancer
}

\author{
JOHJI HARA, HIROSHI MIYATA, MAKOTO YAMASAKI, KEIJIRO SUGIMURA, \\ TSUYOSHI TAKAHASHI, YUKINORI KUROKAWA, KIYOKAZU NAKAJIMA, \\ SHUJI TAKIGUCHI, MASAKI MORI and YUICHIRO DOKI
}

Department of Gastroenterological Surgery, Graduate School of Medicine, Osaka University, Osaka 565-0871, Japan

Received March 14, 2012; Accepted May 14, 2012

DOI: $10.3892 /$ or.2013.2876

\begin{abstract}
The relationship between the epithelial-mesenchymal transition (EMT) and resistance to anticancer treatment has attracted attention in recent years. However, to date, there is no direct clinical evidence for a link between the mesenchymal phenotype and chemoresistance in human malignancies. The expression of EMT-related markers, including E-cadherin, Snail, vimentin, ZEB1, $\beta$-catenin and $\mathrm{N}$-cadherin was examined immunohistochemically in 185 tissue samples from patients with esophageal cancer (including 93 patients who received preoperative chemotherapy followed by surgery and 92 patients who underwent surgery without preoperative therapy). The relationship between the expression of the above markers and clinical outcome including prognosis and response to chemotherapy was also examined. The expression of E-cadherin, a marker of epithelial cells, was significantly lower in residual tumors than chemo-naive tumors $(\mathrm{P}=0.003)$. The expression of Snail $(\mathrm{P}=0.028)$, ZEB1 $(\mathrm{P}<0.001)$ and $\mathrm{N}$-cadherin $(\mathrm{P}=0.001)$, markers of mesenchymal cells, was higher in residual tumors than in chemonaive tumors. The expression of E-cadherin correlated inversely with that of Snail $(\mathrm{P}<0.001)$. Reduced expression of E-cadherin and increased expression of Snail in residual tumors from patients who received chemotherapy correlated significantly with poor response to chemotherapy and short survival time. Multivariate analysis identified Snail expression as an independent prognostic factor, along with tumor depth, in patients who received preoperative chemotherapy for esophageal cancer. The results suggest transition of residual esophageal cancer cells to mesenchymal phenotype after chemotherapy
\end{abstract}

Correspondence to: Dr Hiroshi Miyata, Department of Gastroenterological Surgery, Osaka University, Graduate School of Medicine, 2-2 Yamadaoka, Suita, Osaka 565-0871, Japan

E-mail: hmiyata@gesurg.med.osaka-u.ac.jp

Key words: esophageal cancer, chemotherapy resistance, epithelialmesenchymal transition, snail, E-cadherin and this contributes to resistance to chemotherapy and poor prognosis in patients with esophageal cancer.

\section{Introduction}

Esophageal cancer is one of the most aggressive and lethal malignancies. Surgical treatment is considered the standard management approach for esophageal cancer. However, despite recent advances in surgical technique, the prognosis of patients who undergo surgery alone is poor (1-3). Thus, multimodal treatment such as surgery following neoadjuvant chemotherapy or chemoradiotherapy is advocated. In fact, several clinical trials have shown that such multimodal therapies prolonged survival of patients with esophageal cancer (4-7). However, the reported response rate to chemotherapy in esophageal cancer is only $19-40 \%(1,2,4,8-10)$ and chemoresistance has emerged as a serious problem. Thus, there is a need to understand the underlying mechanism of chemoresistance in esophageal cancer.

Epithelial-mesenchymal transition (EMT) is a biologic process that allows a polarized epithelial cell, which normally interacts with the basement membrane via its basal surface, to undergo multiple biochemical changes that enable it to assume a mesenchymal phenotype. The latter phenotype is characterized by enhanced migratory capacity, invasiveness, high resistance to apoptosis and enhanced production of components of the extracellular matrix (ECM) (11). EMT and the reverse process, termed mesenchymal-epithelial transition (MET), play a central role in embryogenesis (type 1 EMT). EMT is also associated with wound healing, tissue regeneration and organ fibrosis (type 2 EMT) (12-14). Moreover, EMT occurs in neoplastic cells that have previously undergone genetic and epigenetic changes, specifically in genes that favor clonal outgrowth and the development of localized tumors (type 3 EMT). Upon undergoing EMT, cancer cells acquire migratory and invasiveness properties that allow them to migrate through the ECM, resulting in increased metastatic potential $(15,16)$.

Accumulating evidence suggests a direct link between EMT and acquisition of stem cell characteristics (17). Induction of EMT confers many of the properties of self-renewing stem cells $(17,18)$. These findings suggest that EMT plays an impor- 
tant role in resistance to chemotherapy, because cancer stem cells are considered responsible for resistance to anticancer treatment, such as chemotherapy and radiotherapy (19-21). A possible association between EMT and chemotherapy resistance is suggested by recent studies on various cancer cells. However, there is virtually no direct clinical evidence that links mesenchymal phenotype to chemoresistance in human malignancies. Moreover, the association between EMT and chemoresistance has not been elucidated in esophageal cancers.

The present study was designed to determine the expression of EMT-related markers, including E-cadherin, snail, ZEB1 and vimentin, in residual tumors after chemotherapy using samples obtained from patients who underwent preoperative chemotherapy for esophageal cancers. The study also investigated the relationship between the expressions of such EMT markers with prognosis of patients who underwent chemotherapy.

\section{Materials and methods}

Patients and tissue samples. The 185 tissue samples were obtained from patients who underwent radical esophagectomy with lymph node dissection for thoracic esophageal cancer between 1999 and 2007 at the Department of Gastroenterological Surgery, Graduate School of Medicine, Osaka University (Osaka, Japan). Informed consent was obtained from each patient prior to participation in the study. Of these patients, 93 received preoperative chemotherapy followed by surgery while the remaining 92 patients underwent surgery without preoperative therapy. In 65 of the 93 patients who underwent preoperative chemotherapy followed by surgery, endoscopic biopsy samples were obtained before treatment and used for immunohistochemical analysis. Two courses of 4-week preoperative chemotherapy with cisplatin at $70 \mathrm{mg} / \mathrm{m}^{2}$, adriamycin at $35 \mathrm{mg} / \mathrm{m}^{2}$ by rapid intravenous infusion on Day 1 and $5-\mathrm{FU}$ at $700 \mathrm{mg} / \mathrm{m}^{2}$ by continuous intravenous infusion on Days 1-7 followed by 3-weeks off were scheduled before surgical treatment $(6,22)$. The median duration of the follow-up period was 46 months (range, 18-78 months). Furthermore, 107 patients (57.8\%) died during the follow-up.

Immunohistochemistry and evaluation. Resected tumor specimens were fixed with $10 \%$ formalin in phosphate-buffered saline (PBS). The paraffin-embedded tissue blocks were sectioned at 4- $\mu \mathrm{m}$ slices. The sections were deparaffinized in xylene and dehydrated in graded ethanol. For antigen retrieval, they were incubated in $10 \mathrm{mM}$ citrate buffer at $95^{\circ} \mathrm{C}$ water bath for $40 \mathrm{~min}$. The endogenous peroxidase activity in the tissue specimens was blocked by incubating the slides in $3 \%$ hydrogen peroxide $\left(\mathrm{H}_{2} \mathrm{O}_{2}\right)$ solution in methanol at room temperature for $20 \mathrm{~min}$. After treatment of the sections with $1 \%$ bovine serum albumin for $30 \mathrm{~min}$ at room temperature to block nonspecific reactions, all sections were incubated with a primary antibody at working dilution in a humidified chamber at $4^{\circ} \mathrm{C}$ overnight. The antibodies used in the study were antiE-cadherin monoclonal antibody (mAb, dilution 1:100, buffer pH 9.0; Dako, Corp., Carpinteria, CA), anti-Snail polyclonal antibody (pAb, dilution 1:100, buffer pH 9.0; Santa Cruz
Biotechnology, Inc., Santa Cruz, CA), anti-vimentin mAb (dilution 1:100, buffer pH 9.0; Santa Cruz Biotechnology, Inc.), anti-ZEB1 mAb (dilution 1:500, buffer pH 6.0; Dako, Corp.), anti- $\beta$-catenin mAb (dilution 1:100, buffer $\mathrm{pH} 9.0$; Dako, Corp.), anti-N-cadherin pAb (dilution 1:200, buffer $\mathrm{pH} 9.0$; Millipore, Bedford, MA). After incubation with secondary antibodies for $20 \mathrm{~min}$, the reactions were visualized using Vectastain ABC immunoperoxidase kit (Vector Laboratories, Burlington, VT) with 3,3'-diaminobenzidine, which stained the antigen brown, and hematoxylin counterstaining.

Two investigators (J.H. and H.M.) independently evaluated the immunohistochemical sections. The deepest invaded area, called the invasive front, was recorded. The degree of E-cadherin and $\beta$-catenin immunostaining was graded as reduced, negative or cytoplasmic immunoreactivity; preserved, strong linear immunoreactivity on the cell membrane (23). The expression levels of nuclear-Snail and cytoplasmic-vimentin, cytoplasmic-ZEB1, membrane- or cytoplasmic-N-cadherin were scored as negative, $\leq 10 \%$ positive tumor cells; positive, $>10 \%$ positive tumor cells (Fig. 1).

Clinical and histopathological evaluation of response to chemotherapy. Two weeks after 2 cycles of neoadjuvant chemotherapy, all patients were re-assessed to evaluate the clinical response to chemotherapy by endoscopy, computed tomography (CT) and positron emission tomography (PET). The World Health Organization response criteria for measureable disease and the criteria of the Japanese Society for Esophageal Diseases were used to assess clinical response $(24,25)$. A complete response (CR) was defined as disappearance of all lesions. A CR of the primary tumor represented disappearance of the tumor on CT scan and/or PET scan and endoscopy. A partial response (PR) was defined as $>50 \%$ reduction in primary tumor size and lymph node metastasis, as confirmed by CT scan. Progressive disease (PD) was defined as $>25 \%$ increase in the primary tumor or the appearance of new lesions. Stable disease (SD) was defined as neither sufficient decrease to qualify for PR nor sufficient increase to qualify for PD.

Based on the percentage of viable residual tumor cells at the primary site after neoadjuvant chemotherapy, curative effect was classified into five categories. Briefly, the percentage of viable residual tumor cells within the entire cancer tissue was assessed as follows: grade 3, no viable residual tumor cells are evident; grade 2, viable residual tumor cells account for less than one-third of tumor tissue; grade $1 \mathrm{~b}$, viable residual tumor cells account for less than one-third or more but less than twothirds of tumor tissue; grade 1a, viable residual tumor cells account for two-thirds or more tumor tissue; and grade 0 , no recognizable histlogical chemotherapy effect $(6,25)$.

Statistical analysis. Statistical analysis of group differences was performed using the $\chi^{2}$ test, Fisher's exact test or MannWhitney U test. For survival analysis, the Kaplan-Meier method was used to assess survival distribution according to EMT-marker expression and differences in survival were estimated using the log-rank test. The Cox proportional hazards regression model was used to analyze the simultaneous influence of prognostic factors. Wilcoxon signed-ranks test was used to assess the change in E-cadherin and Snail expression 

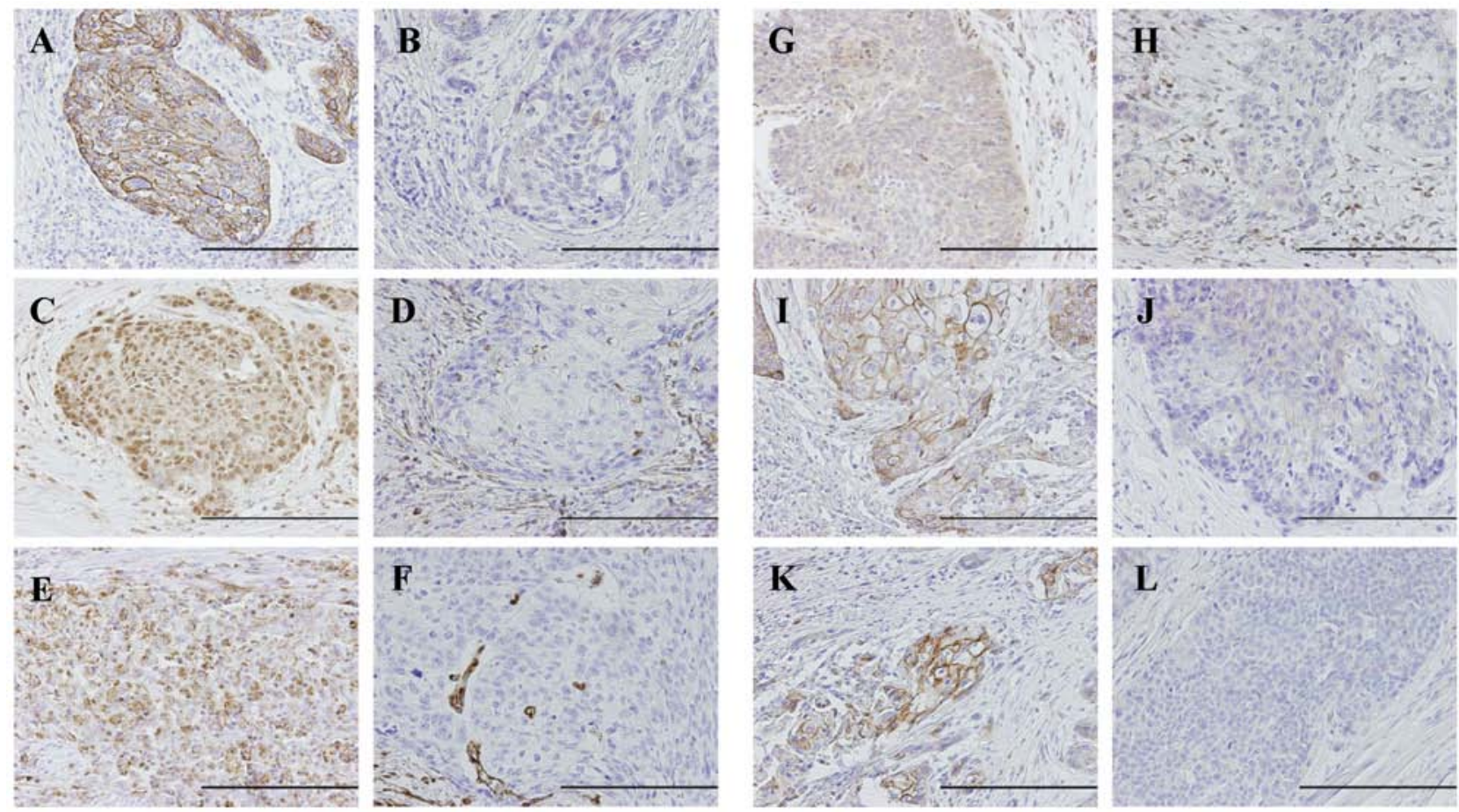

Figure 1. Immunohistochemical expression of E-cadherin, Snail, vimentin, ZEB1, $\beta$-catenin and N-cadherin in human esophageal squamous cell carcinoma. We examined the deepest invading area, known as the invasive front. (A) Membranous expression of E-cadherin. (B) Cytoplasmic and negative expression of E-cadherin. (C) Nuclear expression of Snail. (D) Negative nuclear expression of Snail. (E) Cytoplasmic expression of vimentin. (F) Lack of expression of vimentin. (G) Cytoplasmic expression of ZEB1. (H) Negative expression of ZEB1. (I) Membranous expression of $\beta$-catenin. (J) Cytoplasmic and negative expression of $\beta$-catenin. (K) Membranous or cytoplasmic expression of $\mathrm{N}$-cadherin. (L) Lack of expression of N-cadherin. Magnification, x200.

Table I. Characteristics of 185 patients with esophageal cancer.

\begin{tabular}{lccr}
\hline & \multicolumn{2}{c}{ Chemotherapy } & \\
\cline { 2 - 3 } & Residual (n=93) & Naive (n=92) & P-value \\
\hline Gender (male/female) & $79 / 14$ & $83 / 9$ & 0.276 \\
Age (mean) & 64.0 & 63.7 & 0.512 \\
Tumor location (upper/middle/lower) & $22 / 36 / 35$ & $12 / 47 / 33$ & 0.236 \\
Differentiation (G1,2/G3,4) & $65 / 28$ & $75 / 17$ & 0.418 \\
Depth of invasion (pT1-2/3-4) & $32 / 61$ & $41 / 51$ & 0.157 \\
Lymph node metastasis (pN0/1) & $27 / 65$ & $33 / 59$ & 0.345 \\
Lymphatic permeation (positive/negative) & $77 / 16$ & $70 / 22$ & 0.258 \\
Venous permeation (positive/negative) & $52 / 41$ & $43 / 49$ & 0.212 \\
\hline
\end{tabular}

after chemotherapy. A P-value of $<0.05$ denoted the presence of statistically significant difference between groups. All statistical analyses were performed using the software package JMP 8 for Windows (SAS Institute, Inc., Cary, NC).

\section{Results}

Expression of EMT makers in residual and chemo-naive tumors. Of the 195 tumors, 93 tumors were residual tumors after preoperative chemotherapy and 92 tumors were chemonaive tumors without preoperative therapy. There was no significant difference between residual tumors and chemonaive tumors in differentiation, tumor depth and lymph node metastasis (Table I).
We quantitated the expression of the epithelial marker E-cadherin and mesenchymal markers snail, vimentin, ZEB1, and $\mathrm{N}$-cadherin in residual tumors and chemo-naive tumors (Table II). Fifty percent (46/92) of chemo-naive tumors stained strongly for E-cadherin, while $71 \%$ of residual tumors stained weakly for E-cadherin. Statistical analysis indicated significant underexpression of E-cadherin, as a marker of epithelial cells, in residual tumors compared with chemo-naive tumors $(\mathrm{P}=0.003)$. Snail expression was significantly higher in residual tumors than in chemo-naive tumors $(\mathrm{P}=0.028)$. Similarly, the expression levels of ZEB1 and N-cadherin were significantly higher in residual tumors than in chemo-naive tumors $(\mathrm{P}<0.001$ and $\mathrm{P}=0.001$, respectively). However, there were no significant differences in the expression levels of vimentin and $\beta$-catenin 
Table II. Expression of mesenchymal and epithelial markers in residual tumors after chemotherapy and chemo-naive tumors.

\begin{tabular}{|c|c|c|c|c|}
\hline & \multicolumn{2}{|c|}{ Chemotherapy } & \multirow[b]{2}{*}{$\begin{array}{c}\text { Total } \\
(n=185)\end{array}$} & \multirow[b]{2}{*}{ P-value } \\
\hline & $\begin{array}{l}\text { Residual } \\
(n=93)\end{array}$ & $\begin{array}{l}\text { Naive } \\
(n=92)\end{array}$ & & \\
\hline \multicolumn{5}{|l|}{ E-cadherin } \\
\hline Preserved & $27(29.0)$ & $46(50.0)$ & $73(39.5)$ & 0.003 \\
\hline Reduced & $66(71.0)$ & $46(50.0)$ & $112(60.1)$ & \\
\hline \multicolumn{5}{|l|}{ Snail } \\
\hline Positive & $66(71.0)$ & $51(55.4)$ & $117(63.4)$ & 0.028 \\
\hline Negative & $27(29.0)$ & $41(44.6)$ & $68(36.6)$ & \\
\hline \multicolumn{5}{|l|}{ Vimentin } \\
\hline Positive & $11(11.8)$ & $8(8.7)$ & $19(10.3)$ & 0.482 \\
\hline Negative & $82(88.2)$ & $84(91.3)$ & $166(89.7)$ & \\
\hline \multicolumn{5}{|l|}{ ZEB1 } \\
\hline Positive & $36(38.7)$ & $14(15.2)$ & $50(27.0)$ & $<0.001$ \\
\hline Negative & $57(61.3)$ & $78(84.8)$ & $135(73.0)$ & \\
\hline \multicolumn{5}{|l|}{$\beta$-catenin } \\
\hline Preserved & $32(34.4)$ & $27(29.3)$ & 59 (31.9) & 0.460 \\
\hline Reduced & $61(65.6)$ & $65(70.1)$ & $126(68.1)$ & \\
\hline \multicolumn{5}{|l|}{$\mathrm{N}$-cadherin } \\
\hline Positive & $51(54.8)$ & $29(31.5)$ & $80(43.2)$ & 0.001 \\
\hline Negative & $42(45.2)$ & $63(68.5)$ & $105(66.8)$ & \\
\hline
\end{tabular}

Data are numbers (percentages) of patients.
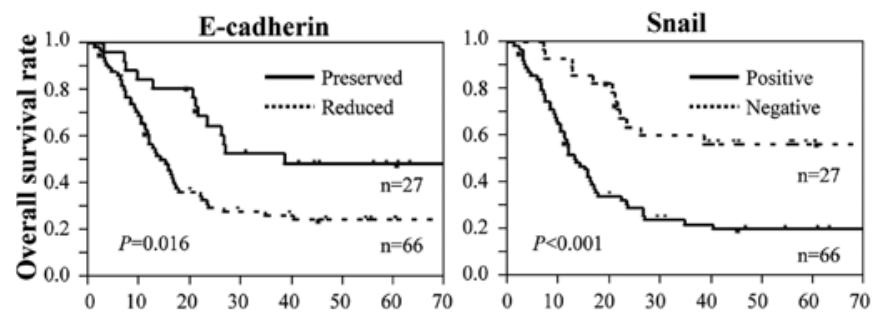

Time after surgery (months)

Figure 2. Postoperative overall survival curves according to the immunohistochemical expression of E-cadherin and Snail in the residual group. Left: reduced expression of E-cadherin correlated significantly with short survival of patients of the residual group. Right: high expression of Snail correlated significantly with short survival of patients of the residual group.

between the two types of tumors. Taken together, higher expression of mesenchymal markers and lower expression of epithelial markers characterize residual tumors after chemotherapy.

We examined the relationship between E-cadherin expression, as an epithelial marker, and the expression of several mesenchymal markers (N-cadherin, vimentin, Snail and ZEB1) in the residual group. E-cadherin expression correlated inversely with Snail expression (Table III).

Relationship between EMT markers and response to chemotherapy. Next, we examined the relationship between the
Table III. Relationship between expression of E-cadherin and EMT markers in the residual group.

\begin{tabular}{lcccc}
\hline & \multicolumn{2}{c}{ E-cadherin } & & \\
\cline { 2 - 3 } & $\begin{array}{c}\text { Preserved } \\
(\mathrm{n}=27)\end{array}$ & $\begin{array}{c}\text { Reduced } \\
(\mathrm{n}=66)\end{array}$ & $\begin{array}{c}\text { Total } \\
(\mathrm{n}=93)\end{array}$ & P-value \\
\hline Snail & & & & \\
Positive & $10(37.0)$ & $56(84.8)$ & $66(71.0)$ & $<0.001$ \\
Negative & $17(63.0)$ & $10(15.2)$ & $27(29.0)$ & \\
Vimentin & & & & \\
Positive & $2(3.7)$ & $9(13.6)$ & $11(11.8)$ & 0.379 \\
Negative & $25(96.3)$ & $57(86.4)$ & $82(88.1)$ & \\
ZEB1 & & & & \\
Positive & $8(29.6)$ & $28(42.4)$ & $36(38.7)$ & 0.245 \\
Negative & $19(70.4)$ & $38(57.6)$ & $57(61.3)$ & \\
$\beta$-catenin & & & & \\
Preserved & $12(44.4)$ & $20(30.3)$ & $32(34.4)$ & 0.197 \\
Reduced & $15(55.6)$ & $46(69.7)$ & $61(65.6)$ & \\
N-cadherin & & & & \\
Positive & $17(63.0)$ & $34(51.5)$ & $51(54.8)$ & 0.311 \\
Negative & $10(27.0)$ & $32(48.5)$ & $42(45.2)$ & \\
\hline
\end{tabular}

Data are numbers (percentages) of patients.

expression of EMT markers and the response to chemotherapy in the residual tumors. With regard to the clinical response, weak E-cadherin expression correlated significantly with clinically poor response (SD/PD), but not with clinically good response $(\mathrm{PR})(\mathrm{P}=0.009$, Table IV). On the other hand, positive staining for Snail expression in tumors correlated significantly with SD/PD, but not PR ( $\mathrm{P}=0.009)$.

Similar to the clinical response, negative E-cadherin expression and positive staining for Snail expression correlated with histopathologically minor response (Grade 0/1a), but not with major response Grade $1 \mathrm{~b} / 2(\mathrm{P}=0.001$ and $\mathrm{P}=0.027$, respectively) (Table V).

Relationship between EMT markers and survival. We also examined relationship between the expression of EMT markers and prognosis of patients who underwent preoperative chemotherapy for esophageal cancer. Low expression of E-cadherin correlated significantly with short survival time (Fig. 2). In contrast, high expression of Snail correlated significantly with short survival time (Fig. 2). Multivariate analysis identified Snail expression as an independent prognostic factor, together with tumor depth, in patients who received preoperative chemotherapy for esophageal cancer (Table V).

Changes in E-cadherin and Snail expression after chemotherapy and survival. In 65 of 93 patients with esophageal cancer who underwent preoperative chemotherapy followed by surgery, we used immunohistochemistry to compare biopsy samples obtained before chemotherapy with the surgical 
Table IV. Relationship between response to chemotherapy and immunohistochemical expression of E-cadherin, Snail, vimentin, ZEB1, $\beta$-catenin and N-cadherin in residual tumors.

\begin{tabular}{|c|c|c|c|c|c|c|c|}
\hline & \multirow[b]{2}{*}{ Total $(n=93)$} & \multicolumn{3}{|c|}{ Clinical response } & \multicolumn{3}{|c|}{ Pathological response } \\
\hline & & $\mathrm{PD} / \mathrm{SD}(\mathrm{n}=47)$ & PR $(n=46)$ & P-value & Grade $0 / 1 \mathrm{a}(\mathrm{n}=67)$ & Grade $1 b / 2(n=26)$ & P-value \\
\hline \multicolumn{8}{|l|}{ E-cadherin } \\
\hline Preserved & $27(29)$ & $8(17)$ & $19(41)$ & 0.009 & $13(19)$ & $14(54)$ & 0.001 \\
\hline Reduced & $66(71)$ & $39(83)$ & $27(59)$ & & $54(81)$ & $12(46)$ & \\
\hline \multicolumn{8}{|l|}{ Snail } \\
\hline Positive & $66(71)$ & $39(83)$ & $27(59)$ & 0.009 & $52(72)$ & $14(54)$ & 0.027 \\
\hline Negative & $27(29)$ & $8(17)$ & $19(41)$ & & $15(22)$ & $12(46)$ & \\
\hline \multicolumn{8}{|l|}{ Vimentin } \\
\hline Positive & $11(12)$ & $5(11)$ & $6(13)$ & 0.719 & $8(12)$ & $3(12)$ & 0.957 \\
\hline Negative & $82(88)$ & $42(89)$ & $40(87)$ & & $59(88)$ & $23(88)$ & \\
\hline \multicolumn{8}{|l|}{ ZEB1 } \\
\hline Positive & $36(39)$ & $15(31)$ & $21(46)$ & 0.173 & $26(39)$ & $10(38)$ & 0.976 \\
\hline Negative & $57(61)$ & $32(68)$ & $25(54)$ & & $41(41)$ & $16(62)$ & \\
\hline \multicolumn{8}{|l|}{$\beta$-catenin } \\
\hline Preserved & $32(34)$ & $17(36)$ & $15(33)$ & 0.717 & $23(34)$ & $9(35)$ & 0.979 \\
\hline Reduced & $61(66)$ & $30(64)$ & $31(67)$ & & $44(66)$ & $17(65)$ & \\
\hline \multicolumn{8}{|l|}{$\mathrm{N}$-cadherin } \\
\hline Positive & $51(55)$ & $28(60)$ & $23(50)$ & 0.353 & $40(60)$ & $11(42)$ & 0.131 \\
\hline Negative & $42(45)$ & $19(40)$ & $23(50)$ & & $27(40)$ & $15(58)$ & \\
\hline
\end{tabular}

Data are numbers (percentages) of patients. PD, progressive disease; SD, stable disease; PR, partial response.

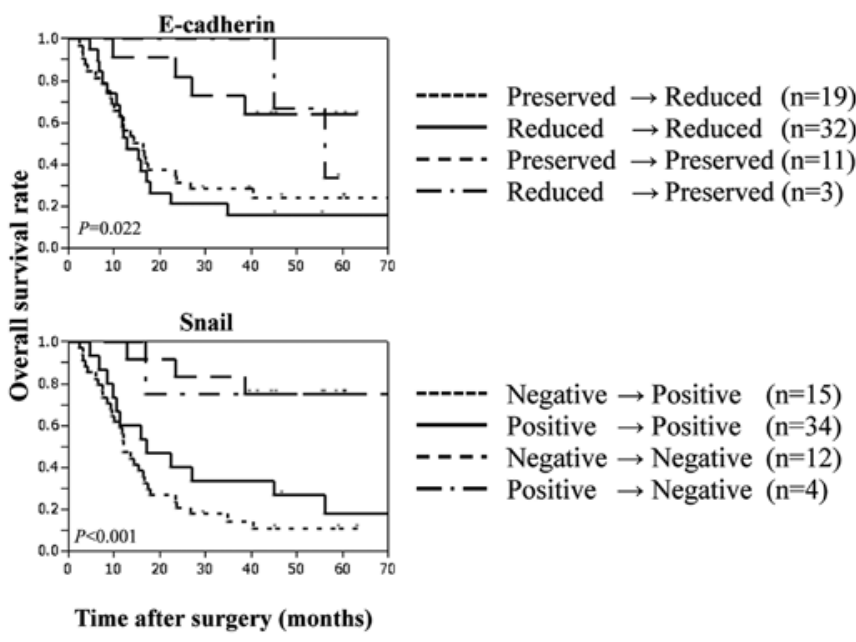

Figure 3. Postoperative overall survival curves according to the immunohistochemical expression of E-cadherin and Snail before and after chemotherapy. Top: short survival of patients $(n=51)$ with decreased expression of E-cadherin after chemotherapy and unchanged low expression of E-cadherin after chemotherapy. Bottom: short survival of patients $(n=49)$ with increased expression of Snail after chemotherapy and unchanged positive expression of Snail after chemotherapy.

specimens after chemotherapy. Among these 65 patients, chemotherapy decreased the expression of E-cadherin in 19 (preserved $\rightarrow$ reduced) (Table VI). The survival time was significantly shorter in 51 patients with low E-cadherin expression [including the above 19 patients and 32 patients who showed no change in their low E-cadherin expression after chemotherapy (reduced $\rightarrow$ reduced)], compared with 11 patients with preserved expression of E-cadherin throughout chemotherapy (Fig. 2). With regard to Snail expression, chemotherapy increased Snail expression in 15 of the 65 patients (negative to positive). The survival time was significantly shorter in 49 patients with positive Snail expression [including the above 15 patients and 34 patients who showed no change in positive Snail expression after chemotherapy (negative to positive)], compared with 12 patients with Snail-negative tumors throughout chemotherapy (Fig. 3).

\section{Discussion}

Although recent evidence indicates that EMT does not only cause increased metastasis but also contributes to chemoresistance, there is no direct clinical evidence for a link between mesenchymal phenotype and chemoresistance in human malignancies. In this study, we examined the expression of EMT-related markers in residual tumors after chemotherapy using samples obtained from patients who underwent preoperative chemotherapy for esophageal cancer. The results showed reduced expression of E-cadherin (a marker of epithelial cells) and increased expression of Snail, ZEB1 and $\mathrm{N}$-cadherin (markers of mesenchymal cells) in residual tumors after chemotherapy, compared with chemo-naive tumors. Moreover, the reduced expression of E-cadherin and increased expression of snail in residual tumors were significantly asso- 
Table V. Univariate and multivariate analyses of prognostic factors.

\begin{tabular}{|c|c|c|c|c|c|c|}
\hline & \multicolumn{3}{|c|}{ Univariate } & \multicolumn{3}{|c|}{ Multivariate } \\
\hline & HR & $95 \% \mathrm{CI}$ & P-value & HR & $95 \% \mathrm{CI}$ & P-value \\
\hline Gender (male/female) & 0.84 & $0.46-1.71$ & 0.619 & & & \\
\hline Age $(\leq 65 />65)$ & 1.22 & $0.74-2.02$ & 0.422 & & & \\
\hline Tumor location (upper, middle/lower) & 0.73 & $0.43-1.21$ & 0.225 & & & \\
\hline Differentiation $(\mathrm{G} 1,2 / \mathrm{G} 3,4)$ & 0.97 & $0.55-1.74$ & 0.920 & & & \\
\hline Depth of invasion (pT1-2/pT3-4) & 2.49 & $1.35-5.05$ & 0.003 & 2.13 & $1.12-4.37$ & 0.018 \\
\hline Lymph node metastasis ( $\mathrm{pN} 0 / 1)$ & 3.12 & $2.32-4.21$ & $<0.001$ & 2.12 & $1.21-4.24$ & 0.009 \\
\hline Lymphatic invasion (positive/negative) & 2.09 & $0.81-3.99$ & 0.181 & & & \\
\hline Venous invasion (positive/negative) & 1.21 & $0.74-2.02$ & 0.437 & & & \\
\hline E-cadherin (preserved/reduced) & 0.56 & $0.30-0.98$ & 0.043 & 1.21 & $0.63-2.21$ & 0.551 \\
\hline Snail (positive/negative) & 3.31 & $1.78-6.71$ & $<0.001$ & 3.83 & $1.96-8.11$ & $<0.0001$ \\
\hline Vimentin (positive/negative) & 0.86 & $0.38-1.70$ & 0.679 & & & \\
\hline ZEB1 (positive/negative) & 0.88 & $0.51-1.45$ & 0.617 & & & \\
\hline$\beta$-catenin (preserved/reduced) & 1.41 & $0.85-2.33$ & 0.179 & & & \\
\hline N-cadherin (positive/negative) & 0.93 & $0.56-1.53$ & 0.760 & & & \\
\hline Clinical response (PD-SD/PR) & 2.29 & $1.38-3.87$ & 0.001 & 1.68 & $0.99-2.92$ & 0.052 \\
\hline
\end{tabular}

$\mathrm{HR}$, hazard ratio; 95\% CI, 95\% confidence interval; PD, progressive disease; SD, stable disease; PR, partial response.

Table VI. Changes in E-cadherin and Snail expression after chemotherapy.

\begin{tabular}{lcccc}
\hline & Pre-CT biopsy & Residual & $\mathrm{n}$ & P-value \\
\hline \multirow{2}{*}{ E-cadherin } & Preserved & Reduced & 19 & $<0.001$ \\
& Reduced & Reduced & 32 & \\
& Preserved & Preserved & 11 & \\
Snail & Reduced & Preserved & 3 & \\
& Negative & Positive & 15 & 0.019 \\
& Positive & Positive & 34 & \\
& Negative & Negative & 12 & \\
& Positive & Negative & 4 & \\
\hline
\end{tabular}

ciated with poor response to chemotherapy and short survival time in patients who underwent preoperative chemotherapy. These results suggest that residual esophageal tumors after chemotherapy display mesenchymal features, resulting in chemoresistance and poor prognosis.

Reduced expression of E-cadherin, which is a central adhesion molecule located at cell-cell adhesion junctions, is one of the characteristics findings during progression of EMT (26). Previous studies demonstrated that the loss of E-cadherin is associated with tumor progression, tumor metastasis and poor clinical outcome in various human carcinomas (27-31). The association of E-cadherin expression and drug sensitivity has been examined in several types of human cancer. In colorectal cancer, E-cadherin was downregulated in oxaliplatin-resistant colorectal cancer (CRC) cells (28). In gemcitabine-resistance pancreatic cancer cells, E-cadherin expression was decreased and nuclear localization of total $\beta$-catenin was increased (30).
While the above studies showed downregulation of E-cadherin in drug-resistant tumor cell lines, there is little or no evidence for the clinical importance of E-cadherin expression in drugresistant human cancers. Using samples from patients who underwent preoperative chemotherapy for esophageal cancers, we demonstrated in this study the importance of E-cadherin underexpression in chemoresistance in human esophageal cancer.

Snail is recognized as a suppressor of E-cadherin expression. Snail represses the transcription of E-cadherin by binding to the E-box elements in the proximal E-cadherin promoter, thereby triggering a complete EMT and resulting in enhanced tumor invasiveness (30). Accumulating evidence suggests the contribution of Snail expression to therapeutic resistance in various cancers $(28-30,33)$. Paclitaxel-resistant ovarian cancer cells showed upregulation of Snail expression, with marked enhancement of metastatic activity, compared with control cells (30). In head and neck cancer, Snail contributes to cisplatin resistance by upregulating excision repair cross complementation group 1 (ERCC1), which plays a key role in nucleotide excision repair and in platinum-induced DNA adducts (33). In the present study, upregulation of Snail was observed in residual tumors after chemotherapy for esophageal cancers and such high expression was significantly associated with poor response to chemotherapy. These results provide direct evidence for the important role of Snail expression in chemoresistance in human esophageal cancer.

In the present study, we examined the relationship between E-cadherin expression, as an epithelial marker, and the expression of several mesenchymal markers (N-cadherin, vimentin, Snail and ZEB1). In recent years, a switch from E-cadherin to $\mathrm{N}$-cadherin has been often used to monitor the progress of EMT during embryonic development and cancer progression (34). In 
our study, although $\mathrm{N}$-cadherin expression was increased in residual tumors, compared with chemo-naive tumors, we could not find significant inverse relationship between E-cadherin and $\mathrm{N}$-cadherin expression. Snail and ZEB1 are well known transcription repressors of E-cadherin $(29,30,32,35)$, and our results showed inverse correlation between E-cadherin and Snail expression, although we could not find a significant correlation between E-cadherin and ZEB1 expression.

Recent studies have indicated that cancer cells undergoing EMT develop resistance to anticancer drugs. However, it has been difficult to establish the role of EMT in chemoresistance in human clinical samples. In the present study, we investigated whether EMT confers resistance to chemotherapy by comparing the expression of EMT-related markers in residual tumors after chemotherapy with that in chemo-naive tumors. A few studies have previously shown the presence of EMT in residual tumors after conventional anti-cancer therapy. One such study demonstrated recently mesenchymal features of tumor cells that had survived conventional treatment, such as chemotherapy and endocrine therapy, in human breast cancer (36). The results of the present study demonstrating mesenchymal features of tumor cells after chemotherapy in esophageal cancer provide further support to the above previous studies.

One important problem in the present study is whether tumor cells with initial mesenchymal phenotype survive the chemotherapy or whether residual tumor cells acquire mesenchymal features during chemotherapy. In this study, we compared the expression of EMT-related markers such as E-cadherin and Snail before and after chemotherapy in the same case, and found in certain cases mesenchymal features in residual tumors after chemotherapy compared with epithelial features before treatment. This finding suggests that residual tumor cells seem to acquire mesenchymal features during chemotherapy. However, the value of immunohistochemistry in accurate assessment of gene expression in biopsy samples is limited, because biopsy samples do not allow accurate estimation of such events in the invasive front of tumors. Recent studies have pointed to link between EMT phenotype and development of cancer stem cells; cancer cells undergoing EMT exhibit characteristic markers of cancer stem cells and properties of cancer stem cells (17). However, other studies have suggested that cancer stem cells from solid tumors are not actually static entities but rather tumor cells that transiently acquire stemness properties depending on the tumor context (37), although the traditional concept of cancer stem cells is a unidirectional hierarchical model. These findings suggest that residual esophageal cancer cells may transiently acquire mesenchymal features to survive during chemotherapy. In support of this notion, one recent study showed that cancer cell populations employ a dynamic strategy in which individual cells transiently assume a reversibly drug-tolerant state to protect the remaining population from eradication by exposure to lethal anti-cancer drugs (38). Further studies are required to ascertain whether esophageal cancer cells transiently acquire mesenchymal features and stemness properties during chemotherapy in human esophageal cancers.

In conclusion, the present study demonstrated decreased expression of E-cadherin and increased expression of Snail, ZEB1 and N-cadherin in residual tumors after chemotherapy in human esophageal cancers, compared with chemo-naive tumors. Moreover, in patients who underwent preoperative chemotherapy, the reduced expression of E-cadherin and increased expression of Snail in residual tumors correlated significantly with poor response to chemotherapy and poor prognosis. These findings suggest that residual tumors after chemotherapy for esophageal cancer switch to mesenchymal phenotype, resulting in chemoresistance and poor clinical outcome.

\section{Acknowledgements}

This study was supported by a Grant-in-Aid for Young Scientists from the Ministry of Education, Culture, Sports, Science and Technology of Japan.

\section{References}

1. Medical Research Council Oesophageal Cancer Working Group, Surgical resection with or without preoperative chemotherapy in oesophageal cancer: a randomised controlled trial. Lancet 359: 1727-1733, 2002.

2. Kelsen DP, Ginsberg R, Pajak TF, Sheahan DG, Gunderson L, Mortimer J, Estes N, Haller DG, Ajani J, Kocha W, et al: Chemotherapy followed by surgery compared with surgery alone for localized esophageal cancer. N Engl J Med 339: 1979-1984, 1998.

3. Gebski V, Burmeister B, Smithers BM, Foo K, Zalcberg J and Simes J: Australasian Gastro-Intestinal Trials Group, Survival benefits from neoadjuvant chemoradiotherapy or chemotherapy in oesophageal carcinoma: a meta-analysis. Lancet Oncol 8: 226-234, 2007.

4. Law S, Fok M, Chow S, Chu KM and Wong J: Preoperative chemotherapy versus surgical therapy alone for squamous cell carcinoma of the esophagus: a prospective randomized trial. J Thorac Cardiovasc Surg 114: 210-217, 1997.

5. Ando N, Iizuka T, Ide H, Ishida K, Shinoda M, Nishimaki T, Takiyama W, Watanabe H, Isono K, Aoyama N, et al: Japan Clinical Oncology Group, Surgery plus chemotherapy compared with surgery alone for localized squamous cell carcinoma of the thoracic esophagus: a Japan Clinical Oncology Group Study JCOG9204. J Clin Oncol 21: 4592-4596, 2003.

6. Yano M, Takachi K, Doki Y, Miyashiro I, Kishi K, Noura S, Eguchi H, Yamada T, Ohue M, Ohigashi H, et al: Preoperative chemotherapy for clinically node-positive patients with squamous cell carcinoma of the esophagus. Dis Esophagus 19: 158-163, 2006.

7. Tepper J, Krasna MJ, Niedzwiecki D, Hollis D, Reed CE, Goldberg R, Kiel K, Willett C, Sugarbaker D and Mayer R: Phase III trial of trimodality therapy with cisplatin, fluorouracil, radiotherapy, and surgery compared with surgery alone for esophageal cancer: CALGB 9781. J Clin Oncol 26: 1086-1092, 2008.

8. Hilgenberg AD, Carey RW, Wilkins EW Jr, Choi NC, Mathisen DJ and Grillo HC: Preoperative chemotherapy, surgical resection, and selective postoperative therapy for squamous cell carcinoma of the esophagus. Ann Thorac Surg 45: 357-363, 1998

9. Ancona E, Ruol A, Santi S, Merigliano S, Sileni VC, Koussis H, Zaninotto G, Bonavina L and Peracchia A: Only pathologic complete response to neoadjuvant chemotherapy improves significantly the long term survival of patients with resectable esophageal squamous cell carcinoma: final report of a randomized, controlled trial of preoperative chemotherapy versus surgery alone. Cancer 191: 2165-2174, 2001.

10. Urschel JD, Vasan H and Blewett CJ: A meta-analysis of randomized controlled trials that compared neoadjuvant chemotherapy and surgery to surgery alone for resectable esophageal cancer. Am J Surg 183: 274-279, 2002.

11. Kalluri R and Weinberg RA: The basics of epithelial-mesenchymal transition. J Clin Invest 119: 1420-1428, 2009.

12. Zeisberg EM, Tarnavski O, Zeisberg M, Dorfman AL, McMullen JR, Gustafsson E, Chandraker A, Yuan X, Pu WT, Roberts $\mathrm{AB}$, et al: Endothelial-to-mesenchymal transition contributes to cardiac fibrosis. Nat Med 13: 952-961, 2007.

13. Zeisberg M, Yang C, Martino M, Duncan MB, Rieder F, Tanjore $\mathrm{H}$ and Kalluri R: Fibroblasts derive from hepatocytes in liver fibrosis via epithelial to mesenchymal transition. J Biol Chem 282: 23337-23347, 2007. 
14. Kim KK, Kugler MC, Wolters PJ, Robillard L, Galvez MG, Brumwell AN, Sheppard D and Chapman HA: Alveolar epithelial cell mesenchymal transition develops in vivo during pulmonary fibrosis and is regulated by the extracellular matrix. Proc Natl Acad Sci USA 103: 13180-13185, 2006.

15. Thiery JP: Epithelial-mesenchymal transitions in tumour progression. Nat Rev Cancer 2: 442-454, 2002.

16. Yang J and Weinberg RA: Epithelial-mesenchymal transition: at the crossroads of development and tumor metastasis. Dev Cell 14: 818-829, 2008.

17. Mani SA, Guo W, Liao MJ, Eaton EN, Ayyanan A, Zhou AY, Brooks M, Reinhard F, Zhang CC, Shipitsin M, et al: The epithelial-mesenchymal transition generates cells with properties of stem cells. Cell 133: 704-715, 2008

18. Biddle A, Liang X, Gammon L, Fazil B, Harper LJ, Emich H, Costea DE and Mackenzie IC: Cancer stem cells in squamous cell carcinoma switch between two distinct phenotypes that are preferentially migratory or proliferative. Cancer Res 71: 5317-5326, 2011.

19. Kurrey NK, Jalgaonkar SP, Joglekar AV, Ghanate AD, Chaskar PD, Doiphode RY and Bapat SA: Snail and slug mediate radioresistance and chemoresistance by antagonizing p53-mediated apoptosis and acquiring a stem-like phenotype in ovarian cancer cells. Stem Cells 27: 2059-2068, 2009.

20. Eyler CE and Rich JN: Survival of the fittest: cancer stem cells in therapeutic resistance and angiogenesis. J Clin Oncol 26: 2839-2845, 2008.

21. Baumann M, Krause M and Hill R: Exploring the role of cancer stem cells in radioresistance. Nat Rev Cancer 8: 545-554, 2008.

22. Miyata H, Yoshioka A, Yamasaki M, Nushijima Y, Takiguchi S, Fujiwara Y, Nishida T, Mano M, Mori M and Doki Y: Tumor budding in tumor invasive front predicts prognosis and survival of patients with esophageal squamous cell carcinomas receiving neoadjuvant chemotherapy. Cancer 115: 3324-3334, 2009.

23. Usami Y, Chiba H, Nakayama F, Ueda J, Matsuda Y, Sawada N, Komori T, Ito A and Yokozaki H: Reduced expression of claudin-7 correlates with invasion and metastasis in squamous cell carcinoma of the esophagus. Hum Pathol 37: 569-577, 2006.

24. Miller AB, Hoogstraten B, Staquet M and Winkler A: Reporting results of cancer treatment. Cancer 47: 207-214, 1981.

25. Japan Esophageal Society: Japanese classification of esophageal cancer, tenth edition: parts II and III. Esophagus 6: 71-94, 2009.

26. Lombaerts M, van Wezel T, Philippo K, Dierssen JW, Zimmerman RM, Oosting J, van Eijk R, Eilers PH, van de Water B, Cornelisse CJ and Cleton-Jansen AM: E-cadherin transcriptional downregulation by promoter methylation but not mutation is related to epithelial-to-mesenchymal transition in breast cancer cell lines. Br J Cancer 94: 661-671, 2006.
27. Shiozaki H, Tahara H, Oka H, Miyata M, Kobayashi K, Tamura S, Iihara K, Doki Y, Hirano S, Takeichi M and Mori T: Expression of immunoreactive E-cadherin adhesion molecules in human cancers. Am J Pathol 139: 17-23, 1999.

28. Yang AD, Fan F, Camp ER, van Buren G, Liu W, Somcio R, Gray MJ, Cheng H, Hoff PM and Ellis LM: Chronic oxaliplatin resistance induces epithelial-to-mesenchymal transition in colorectal cancer cell lines. Clin Cancer Res 12: 4147-4153, 2006.

29. Kajiyama H, Shibata K, Terauchi M, Yamashita M, Ino K, Nawa A and Kikkawa F: Chemoresistance to paclitaxel induces epithelial-mesenchymal transition and enhances metastatic potential for epithelial ovarian carcinoma cells. Int J Oncol 31: 277-283, 2007.

30. Shah AN, Summy JM, Zhang J, Park SI, Parikh NU and Gallick GE: Development and characterization of gemcitabineresistant pancreatic tumor cells. Ann Surg Oncol 14: 3629-3637, 2007.

31. Arumugam T, Ramachandran V, Fournier KF, Wang H, Marquis L, Abbruzzese JL, Gallick GE, Logsdon CD, McConkey DJ and Choi W: Epithelial to mesenchymal transition contributes to drug resistance in pancreatic cancer. Cancer Res 69: 5820$5828,2009$.

32. Peinado H, Olmeda D and Cano A: Snail, Zeb and bHLH factors in tumour progression: an alliance against the epithelial phenotype? Nat Rev Cancer 7: 415-428, 2007.

33. Hsu DS, Lan HY, Huang CH, Tai SK, Chang SY, Tsai TL, Chang CC, Tzeng CH, Wu KJ, Kao JY and Yang MH: Regulation of excision repair cross-complementation group 1 by Snail contributes to cisplatin resistance in head and neck cancer. Clin Cancer Res 16: 4561-4571, 2010.

34. Hirohashi S and Kanai Y: Cell adhesion system and human cancer morphogenesis. Cell Sci 94: 575-581, 2003.

35. Nieto MA: The snail superfamily of zinc-finger transcription factors. Nat Rev Mol Cell Biol 3: 155-166, 2002

36. Creighton CJ, Li X, Landis M, Dixon JM, Neumeister VM, Sjolund A, Rimm DL, Wong H, Rodriguez A, Herschkowitz JI, et al: Residual breast cancers after conventional therapy display mesenchymal as well as tumor-initiating features. Proc Natl Acad Sci USA 106: 13820-13825, 2009.

37. Roesch A, Fukunaga-Kalabis M, Schmidt EC, Zabierowski SE, Brafford PA, Vultur A, Basu D, Gimotty P, Vogt T and Herlyn M: A temporarily distinct subpopulation of slow-cycling melanoma cells is required for continuous tumor growth. Cell 141: 583-594, 2010.

38. Sharma SV, Lee DY, Li B, Quinlan MP, Takahashi F, Maheswaran S, McDermott U, Azizian N, Zou L, Fischbach MA, et al: A chromatin-mediated reversible drug-tolerant state in cancer cell subpopulations. Cell 141: 69-80, 2010. 